\title{
Design and research of corn combine on-line monitoring system
}

\author{
Wu Mingqing ${ }^{1}$, Shi Jinshui ${ }^{1}$, Wu Changzhong ${ }^{2}$ \\ ${ }^{1}$ Shandong Transport Vocational College, Shandong Weifang, 261206, China; \\ ${ }^{2}$ University of Jinan, Jiwei Road 106, Jinan City, Shandong Province, 250022, China.
}

Keywords: Corn combine; online monitoring system; design and research

\begin{abstract}
In order to improve the automation level of combine, we should pay attention to the realtime detection of combine work. The key components of the machine have a direct impact on the working state of combine. We need to design an online detection system to detect the working condition of components and oil consumption under the working state. The structure design of online monitoring system includes automatic control module, operation parameter acquisition module, human-computer interaction module, GPS positioning module, manual automatic switching module and online transmission module. Through the design of online monitoring system software, the real-time monitoring of working state is realized, which greatly improves the reliability and efficiency.
\end{abstract}

In recent years, agricultural technology has been widely developed. Corn combine harvester plays an important role in the process of harvesting corn. If it breaks down, it will not only waste the efficiency of the machine, but also cause corresponding economic losses. At this time, we should pay attention to the application of online monitoring system.

\section{Performance requirements of corn combined harvest monitoring system}

1) Strong practicability: it can be applied to different models of corn combine, and also can realize the application of detection system comprehensively.

2) Reliability: in the face of severe production environment, the detection system can detect the working parameters of each link and component of the working corn harvester

3) Intellectualization: in the process of testing, collect the parameters of corn combine, analyze the testing contents comprehensively, find out the problems from the analysis, and judge the working state of the machine.

4) Humanization: visual interface and audible and visual alarm can be added to the cab.

\section{Main factors affecting the working performance of combine}

There are many reasons that affect the working state of combine, among which the key parts of the machine have a direct impact on the working state of combine. The surrounding environment and human factors will also have an impact. For example, the production environment and situation of crops and the types of crops are unpredictable factors. For example, the rotation speed and torque of the threshing drum and the walking speed of the machine can be controlled by human.

\subsection{Power consumption of threshing drum}

The research shows that the relationship between the threshing roller device and the feeding quantity of the machine is in direct proportion, which can account for about one third of the total power. The rotation speed and torque of threshing drum are two processes of consumption. When the rotation speed of the drum is increased, the striking force of the Striped bar on the drum will increase, and the frequency and amplitude of the encounter between the Striped bar and the concave grid will increase, and the frequency and amplitude of the forced vibration and self-excited vibration of the logistics will be increased. At this time, due to the increase of the extension dislocation of the crop, it will produce The separation of raw grains. It can be seen that if the 
rotating speed is too large, it will strengthen the role of combing and rubbing, thus promoting the rate of falling off, and will make straw seriously damaged, thus increasing the difficulty of cleaning seeds. In the process of threshing, the friction between the cylinder and the intaglio or the acceleration of antiques in the cylinder will affect the torque. There are also uncontrollable factors, such as the stalk length of threshing crops, the influence of different varieties, humidity and feeding methods. The torque of drum will be affected by the design of concave and bar.

\subsection{Feeding quantity}

The combine has a dynamic system with complex output variables and multi input characteristics. In the process of harvesting crops, the height, planting density, species, water content and forward speed of the combine will affect the change of the feeding amount. In the process of working, the driver should carry out the work according to the previous experience, and can realize the artificial control of the speed through human control and observation, so as to ensure that the combine is in an optimal working state as far as possible. At this time, it needs to be noted that too much or too little feeding is not the best standard. If the feeding is too large, it will cause the pressure on the harvester to increase, and it is easy to block the threshing roller, and it may even cause machine failure and economic loss. If the feeding quantity is too small, the harvester will not play its full role and waste. The detection of crop feeding quantity plays an important role in the working state of combine harvester. When the three standards of harvesting speed, cutting width and stubble height change, the crop feeding quantity will also change, it is necessary to strengthen the research on the performance of detection of crop feeding quantity.

\subsection{Machine forward speed}

Area, ground condition and water content will affect the operation speed of the machine. In the process of combine working, it is not allowed to choose too high working speed. If the speed is too high, there will be leakage, and the increase of feeding amount will also increase the pressure of threshing roller, or even the phenomenon of blockage may occur. The reasonable use of combine can be realized by choosing the right forward speed.

\section{Overall design scheme of monitoring system}

In the process of combine, the driver can't work by simple auditory judgment. At this time, the function of on-line detection system is needed to detect the working condition of components, oil consumption and corn in the working state. The real-time detection of these steps will give a warning and let the driver know the working state in the first time.

From the analysis of the current situation of combine detection system at home and abroad, it can be seen that at present, it is successful to add detection and control sensors on the original basis, and integrate into the working state and characteristics of combine. Through the effective combination of sensors and information technology, we can develop a detection system for detecting the key parameters of combine, and through these parameters, we can be clear The amount of feed. The monitoring system diagram is shown in Figure 1:

\subsection{Design requirements of online monitoring system}

(1) Collection and storage of monitoring data. Through the design of the sensor plate and positioning plate, the rotation speed sensor can collect the rotation speed signal of the combine, and transmit it to the controller through the processing and conditioning circuit. The positioning module transmits the collected geographic location information to the controller through the RS-232 serial port. After receiving the data, the controller processes and stores it in the SD card.

(2) Human computer interface design. This design is more humanized and reasonable. The controller in the combine online monitoring system can communicate with the touch screen through the serial port, and display the collected data on the touch screen.

(3) Fault diagnosis and alarm. Through fault diagnosis and alarm, the driver can find out whether the rotation speed signal of the harvester is normal at the first time, and can also judge the 
occurrence of some component faults. When the fault occurs, it will send a warning through the detection system.

(4) Working mode of the system. Switching between control modes is by manual automatic switching module. When the harvester is in the manual control mode, the operator shall adjust the working speed according to the operating experience and the speed displayed on the touch screen. In the state of automatic mode, the speed can be adjusted automatically by the control of forward speed by online detection system.

(5) Online data transmission. Through the cm8150epwcdma3gdtu wireless data transmission module, the data transmission between the controller and the upper computer is realized. After receiving the transmission data, the monitoring center can determine the working status and position of the harvester in time, and summarize the data of the same type of harvester.

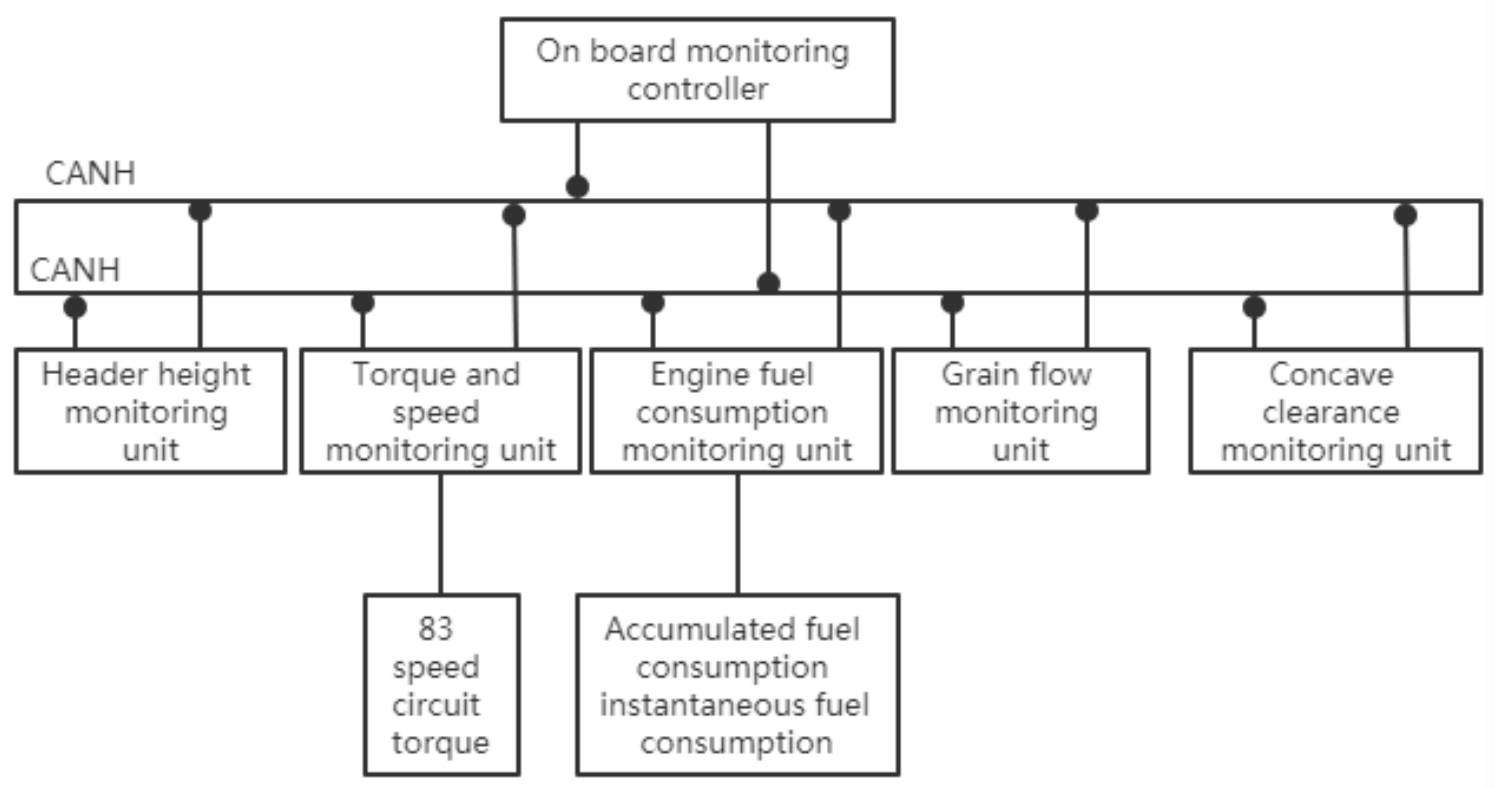

Figure 1 Schematic diagram of automatic monitoring system of combine

\subsection{Structure design of online monitoring system}

According to the design requirements of the online monitoring system, the overall structure of the online monitoring system is designed as shown in Figure 1. The whole online monitoring system is divided into automatic control module, job parameter acquisition module, humancomputer interaction module, GPS positioning module, manual automatic switching module and online transmission module.

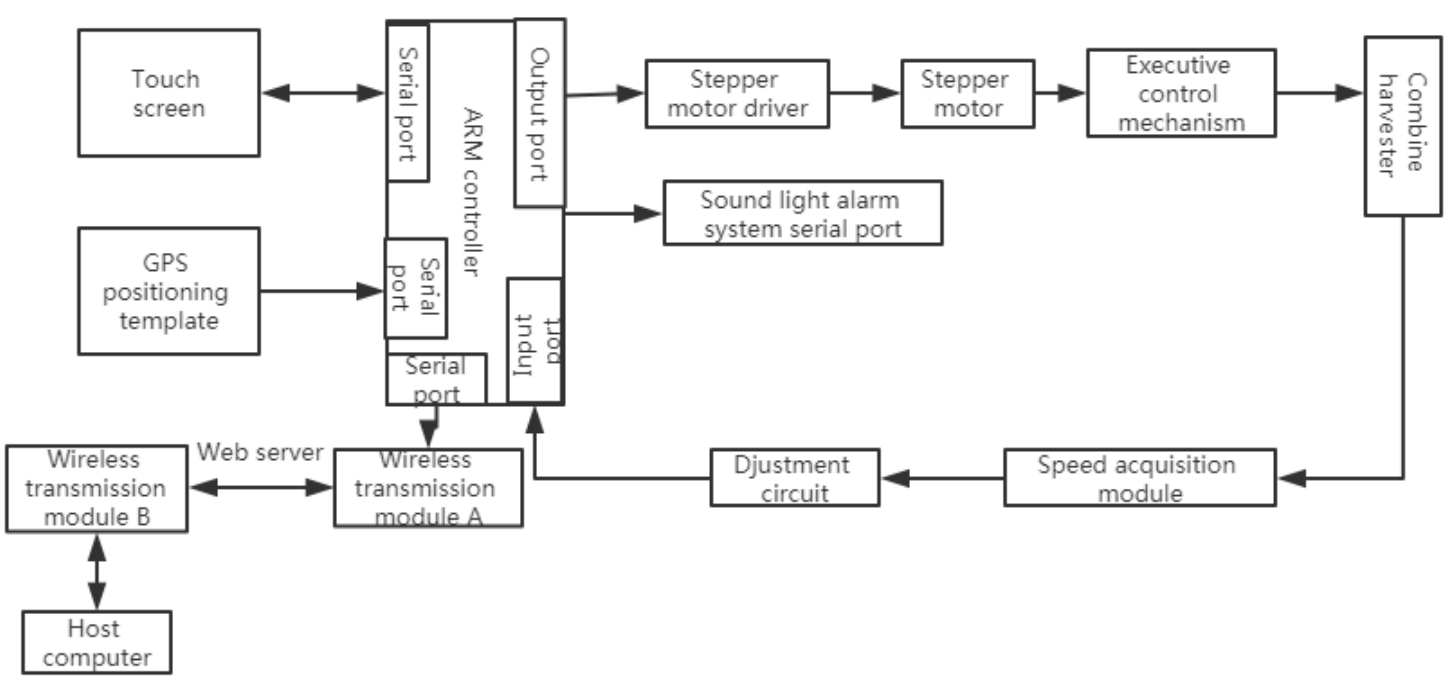

Figure 2 Structure of online monitoring system 
It can be seen from Figure 2 that the speed acquisition module can collect the threshing drum, forward speed, grain conveying auger, conveying tank speed signals, etc. of the combine under the working state. After the signals are collected, they are filtered by the regulation circuit and then transmitted to the arm controller in the input port. After the controller processes the data, they are displayed in the touch screen after the transmission, and after this time Data should be stored during the process. In the process of manual control, the operator can control the working speed. In the automatic control, according to the collected speed and the forward speed, the automatic control algorithm controls the stepper motor driver to control the forward and reverse rotation of the stepper motor to realize acceleration and deceleration. The arm controller transmits the data in the controller to the wireless transmission module a through the serial port communication, and the module $\mathrm{B}$ communicates with the upper computer through the serial port. The two modules use the web server network to transmit the wireless data. The system can realize the fault alarm function on the basis of the traditional data collection, and also realize the timely data transmission with the monitoring center It has played an effective role in online detection.

\section{Software design of online monitoring system}

\subsection{Touch screen software design}

The design of touch screen software includes two aspects. The first aspect is the interface design, including text display, input and output area configuration, alarm indication configuration, function key configuration and other designs. In the specific design, it should also be changed according to the actual needs. The touch screen interface is designed with gtdesigner software. The functions of this software are easy to operate and complete. The system can use this software to design the touch screen parameter setting, parameter display and other interfaces. And this software also includes indicator light, dynamic icon, curve chart and other required tools. The second aspect is the design of the key, which establishes the connection between the configuration function of the touch screen and the I / O port or storage unit relative to the controller, sets the parameters of the controller program on the touch screen, and displays the fault alarm point in the touch screen after the controller, and communicates between the controller and the touch screen through the RS-232 serial port.

\subsection{Automatic control algorithm design}

The change of the forward speed can be adjusted by the step motor and the swing bar, and the manual speed regulating handle and the hydraulic stepless transmission are under the automatic control state. The method adopted in this paper is to identify the automatic control algorithm based on the speed difference mode. It is to combine the calculated actual speed difference with the characteristic mode to call the stepping motor control algorithm under different modes, so as to achieve the purpose of controlling the forward speed by adjusting the speed regulating handle through the forward and reverse rotation of the stepping motor.

The establishment process of the feature mode set is as follows: set the threshing drum speed of the combine as VGT, and set $0.9 \mathrm{vgt}$ and $0.8 \mathrm{vgt}$ as judgment points to divide the corresponding judgment interval as the corresponding feature mode. Set the difference between the speed setting value and $0.9 \mathrm{vgt}$ as $\Delta \mathrm{V} 1$; the difference between the speed setting value and $0.8 \mathrm{vgt}$ as $\Delta \mathrm{V} 2$; the difference between the speed setting value and the actual speed as $\Delta \mathrm{v}$. According to the relationship between $\Delta \mathrm{V}$ and the speed difference, set the following modes: judge the size between the set value and the actual value, set mode 1 when the actual speed is greater than the set value; set mode 2 between 0 and $\Delta \mathrm{V} 1$ when the set value is greater than the actual value; set mode 3 between $\Delta \mathrm{V} 1$ and $\Delta \mathrm{V} 2$; set mode $4>\Delta \mathrm{v} 2$. It can be found from the previous basis and the working state of the combine that when the mode is at 1 , the forward speed is relatively small, and there is no fault, and by judging whether the stepping motor is in the maximum acceleration position, if there is a step motor is in a gradually stable threshing roller speed, and it is a normal harvesting mode, at this time, the roller has no overload, such as If not, the stepper motor will rotate forward rapidly to accelerate 
the combine; if it is in mode 2 state, judge whether the stepper motor is in the maximum position, if so, the stepper motor will stabilize the current state, if not, determine whether the speed is in the rising trend, if so, the stepper motor will rotate forward to accelerate the combine, if not, the stepper motor will rotate backward slowly to slow down the combine When it is in mode 3, it means that the harvester is blocked, but it is not serious. At this time, it should first determine whether the stepping motor is at the minimum operating speed position. If it is, it is required to keep the stepping motor unchanged. If not, it is required to see whether it is in the rising trend. If it is, it is required to keep the current state of the stepping motor. If not, it is required to slowly reverse the stepping motor to realize the slow deceleration of the combine. When it is in mode At 4 o'clock, the combine is blocked and an alarm is given. At this time, when the operation speed is judged as 0 or not, the speed tends to rise. Then the stepping motor keeps the current state. Otherwise, the fast reversal of the stepping motor can realize the fast deceleration of the combine. This algorithm can carry out all-round judgment according to the set feature mode set, which can greatly save the judgment time and improve the response speed.

\section{Conclusion}

Combine harvester can liberate labor force and increase production efficiency in agricultural production, but there are still many deficiencies due to the late development of domestic, so it is necessary to strengthen the research of online monitoring direction to achieve more efficient development of combine harvester.

\section{Acknowledgments}

The project is funded by the "Shandong University Research Program (J18KA045) in 2018".

\section{References}

[1] Liu Guangyin. Research on fault monitoring and diagnosis system of combine harvester [D]. Hubei University of technology, 2019.

[2] Zhang Guangyue, Jin Chengqian, Yang Tengxiang, Liu Zheng, Chen man, Zhou Dongdong. Design and implementation of loss rate monitoring system for combine cleaning [J]. China Journal of agricultural machinery chemistry, 2019,40 (04): 146-150.

[3] Zhang Yawei. Study on mechanism and control strategy of threshing and separation quality of combine harvester [D]. China Agricultural University, 2019.

[4] Zhou Ying. Design of wireless monitoring system for combine working condition [D]. Hubei University of technology, 2018.

[5] Lu La. Research on on-line monitoring system of combine field operation state [D]. Chongqing University of technology, 2018. 